\title{
Zur Debatte um die Reform der gesetzlichen Voraussetzungen einer Unterbringung in der Entziehungsanstalt
}

\author{
Norbert Schalast ${ }^{1}$ \\ Eingegangen: 4. Februar 2021 / Angenommen: 7. Februar 2021 / Online publiziert: 11. März 2021 \\ (c) Der/die Autor(en) 2021
}

\section{Zusammenfassung}

Wie auch durch Müller (2019) vor einiger Zeit in dieser Zeitschrift wird in diesem Beitrag die Forderung nach einer Justierung der rechtlichen Rahmenbedingungen für die Unterbringung in einer Entziehungsanstalt erhoben. Nur begrenzte Übereinstimmung besteht jedoch hinsichtlich Begründung und Ausgestaltung der gebotenen Reform. Die Tendenz zu einem erheblichen Konsum von Rauschmitteln ist bei Straffälligen sehr verbreitet. Die aktuelle Rechtslage, die maßgeblich mit Reformgesetzen in 2007 und 2016 weiterentwickelt wurde, nötigt Angeklagte bei höherer Straferwartung geradezu, ihre Einlassung auf das Ziel der Unterbringung abzustellen, da ein wesentlich kürzerer Freiheitsentzug in Aussicht steht. Gerichte haben einen geringen Ermessensspielraum hinsichtlich der Anordnung der Unterbringung, wenn diese nach Lage der Dinge in Betracht kommt. Trotz Kapazitätserweiterungen stehen die Einrichtungen und Träger des Maßregelvollzugs unter wachsendem Druck. Es wird u. a. begründet, warum die - gutachterlich erforderliche - Prognose der voraussichtlichen Behandlungsdauer sich im ursprünglichen Rahmen von 2 Jahren bewegen sollte. Vor allem aber bedarf es im Hinblick auf die Anordnung der Unterbringung eines realen richterlichen Ermessensspielraums, auf den die Ausgestaltung des $§ 64$ StGB als Sollregelung im Jahre 2007 eigentlich abzielte.

Schlüsselwörter Maßregelvollzug gemäß $§ 64 \mathrm{StGB} \cdot$ Richterlicher Ermessensspielraum $\cdot$ Hang $\cdot$ Erfolgsaussicht . Strafrabatt

\section{Forensic addiction treatment in Germany—the need of readjustment of the legal framework}

\begin{abstract}
In Germany offenders can be sentenced to forensic addiction treatment, which is executed in secure mental hospitals (according to $\S 64$ of the Penal Code). As a rule the persons concerned are transferred to the treatment institution after having served part of their prison sentence. The institutions are under great pressure as a growing number of persons receive a $\S 64$ sentence. The measure is especially attractive for offenders with a relatively long prison sentence as a much earlier discharge from the special hospital may be expected than from prison. Courts have little discretion to apply the $\S 64$ rule if certain presuppositions appear to be fulfilled. The measure is effective and reduces reoffending in comparison to prison sentences alone; however, in roughly $40 \%$ of cases the treatment is discontinued and patients are returned to prison. The $\$ 64$ institutions are a valuable resource but in accordance with Müller (2019) it is thought to lessen the tremendous advantages connected to the measure regarding the length of confinement. Courts should be given more leeway to make use of the measure or not.
\end{abstract}

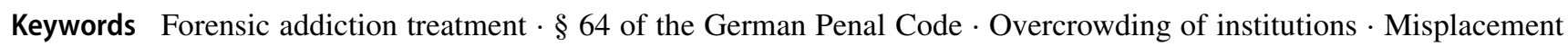
of patients $\cdot$ Small discretionary power of courts

Dipl.-Psychologe Norbert Schalast

norbert.schalast@uni-due.de

1 Institut für forensische Psychiatrie, LVR-Klinikum Essen,

Universität Duisburg-Essen, Postfach 103043, 45030 Essen,

Deutschland 


\section{Zur Situation des § 64-Maßregelvollzugs}

Müller (2019) hat sich vor gut einem Jahr in dieser Zeitschrift über „Ansätze zur Reform der Unterbringung in einer Entziehungsanstalt" geäußert. In seinem Aufsatz (S. 264) stellte er zunächst fest, dass sich die Zahl der forensischen Suchtpatienten von etwa $1000 \mathrm{im}$ Jahr 1990 auf 4000 in 2014 gesteigert, also vervierfacht, habe. Dies stimmt nicht mit den Angaben in der vom Verfasser übernommenen Grafik und den entsprechenden Daten des Statistischen Bundesamtes ${ }^{1}$ überein. Die lauten 1160 (1990) und 3819 (2014), was einer Steigerung um den Faktor 3,3 entspricht (Belegungszahlen für den Stichtag 31.03.). Dabei geht ein Teil dieses Anstiegs - über 200 Fälle - auf die Einbeziehung der neuen Bundesländer in die Strafverfolgungsstatistik im Jahre 2007 zurück. Ähnliches gilt übrigens für die ,Standards für die Behandlung im Maßregelvollzug“ der DGPPN (Müller et al. 2017). Da hieß es, die Einweisungen in die Entziehungsanstalt seien von 1999 bis 2014 auf das Dreifache angestiegen (Müller et al. 2017, S23). Hier betrug der Steigerungsfaktor tatsächlich 2,4, ebenfalls noch nicht bereinigt um die Integration der neuen Bundesländer.

Müller (2019) beschreibt die Zunahme der Patientenzahlen zu Recht als ein Problem für die Einrichtungen und die Kostenträger und schätzt die jährlichen Kosten des § 64Maßregelvollzugs auf über 400 Mio. $€$. Relativierend ist anzumerken, dass die bundesweiten Kosten des Strafvollzugs sich schon im Jahr 2010 auf über 2,8 Mrd. $€$ beliefen. ${ }^{2}$ Die Bewertung des Mehraufwandes für den § 64-Maßregelvollzug bemisst sich daran, ob er seinen Zweck erfüllt, nämlich die Wahrscheinlichkeit von Wiederholungsdelikten und weiteren Haftaufenthalten zu senken (Schalast et al. 2019).

Weiter weist Müller auf die Schwierigkeiten hin, für die Entziehungsanstalten geeignetes Fachpersonal - Ärztinnen, Therapeuten und Pflegekräfte - zu rekrutieren. Dem ist zuzustimmen, wobei die regionalen Verhältnisse diesbezüglich erheblich divergieren. Zudem muss an die noch weit gravierenderen einschlägigen Probleme des Strafvollzugs erinnert werden, die dort - bei beträchtlichem Bedarf keine ausreichende medizinische, psychiatrische und psychotherapeutische Versorgung der Gefangenen ermöglichen (Lehmann 2019).

Müller (S. 264) beklagt die ,zunehmende dissoziale Orientierung und Bereitschaft zum Regelverstoß“ unter den Patienten. „Spektakuläre und öffentlichkeitswirksame Zwischenfälle und Ausbrüche finden sich überwiegend in den

\footnotetext{
${ }^{1}$ Fachserie 10 Reihe 4.1 2013, erschienen 2014.

2 Auskunft des Bundesministeriums für Justiz und Verbraucherschutz im Jahr 2011. Online-Quelle: https://fragdenstaat.de/anfrage/ inhaftierungskosten-in-justizvollzugsanstalten/\#nachricht-763. [Zugegriffen: 24. März 2020].
}

Entziehungsanstalten." Dem ist entgegenzuhalten, dass gerade die Häufigkeiten von Lockerungsmissbräuchen und von Entweichungen aus Kliniken im Laufe der letzten Jahrzehnte geradezu dramatisch zurückgegangen sind (Abb. 1). Der Verfasser (Schalast 1994) hat in einem Projekt die Behandlungsverläufe des Aufnahmejahrgangs 1989 in NRW evaluiert. 111 der insgesamt 136 Patienten fielen im Laufe der Unterbringung durch Suchtmittelkonsum auf. Nur ein Drittel der Patienten entwich nie aus der Unterbringung, nach der Hälfte des Kollektivs wurde insgesamt über 300mal gefahndet. Dies hat seinerzeit wenig Aufregung verursacht. Es wurde mehr oder weniger hingenommen, dass es sich um eine recht instabile Patientengruppe handelte.

Inzwischen hat sich die Häufigkeit entsprechender Vorkommnisse bei etwa 3\% je Patient und Unterbringungsjahr stabilisiert. Es muss hervorgehoben werden, dass es sich dabei eben überwiegend um ein Ausbleiben nach gewährten Lockerungen handelt. Würden die Verhältnisse der 1980er-Jahre in Abb. 1 enthalten sein, so müsste die Ordinate bis 3,0 hinaufreichen.

Erst als Entweichungen ein seltenes Ereignis geworden waren, fanden diejenigen, die noch stattfanden, zunehmende Beachtung. Dabei spielten auch spektakuläre Vorfälle eine Rolle, die den § 63-Maßregelvollzug betrafen und Medien und Öffentlichkeit sensibilisiert hatten. Nicht unerwähnt bleiben darf die tiefgreifende Änderung der Medienlandschaft. Der Maßregelvollzug mit seiner speziellen Klientel ist immer gut für Material ,im gnadenlosen Kampf um die Quote" (Gerhard Mauz; Schalast 1999).

Die Patienten haben von der Stabilisierung des klinischen Alltags profitiert. Unter den chaotischen Bedingungen der 1980er-Jahre kamen sie nicht zur Ruhe, erreichten viele auch vorübergehend kaum Suchtmittelfreiheit. Die Stabilisierung der Entziehungsanstalten resultierte einerseits aus einem höheren Sicherungsgrad: Lockerungsentscheidungen werden sorgfältig abgewogen und Lockerungen später gewährt als in den 1980er-Jahren. Es entstanden aber auch differenziertere Behandlungskonzepte. Viele Kliniken steigerten ihre Kompetenz in der Arbeit mit der schwierigen Patientengruppe. Die Belastung durch internen Drogenkonsum wurde geringer, gerade auch im Vergleich zum Strafvollzug, wenngleich die Stationen - wie alle Einrichtungen für Suchtpatienten - natürlich nicht konstant und durchgängig drogenfrei sind (Zimprichová 2019).

Müller (2019, S. 264f.) kritisiert die rechtlichen Bestimmungen dahingehend, dass weder eine klinische Abhängigkeitsproblematik nach den Diagnosemanualen gefordert wird, noch eine konkrete, zwingende Kausalbeziehung zwischen Sucht und Delinquenz. Dies habe zur Folge, dass Menschen mit primär delinquenter Orientierung in die Unterbringung gebracht würden, bei denen der Drogenhandel eine einträgliche Erwerbsquelle war. Solche Patienten för- 
Abb. 1 Häufigkeit von Lockerungsmissbrauch (Nichtrückkehr, Entweichung, Ausbruch) je Jahr und Patient. Quelle: Stichtagserhebung von der Haar (2016). Keine Datenpunkte für 2010, 2011, 2013

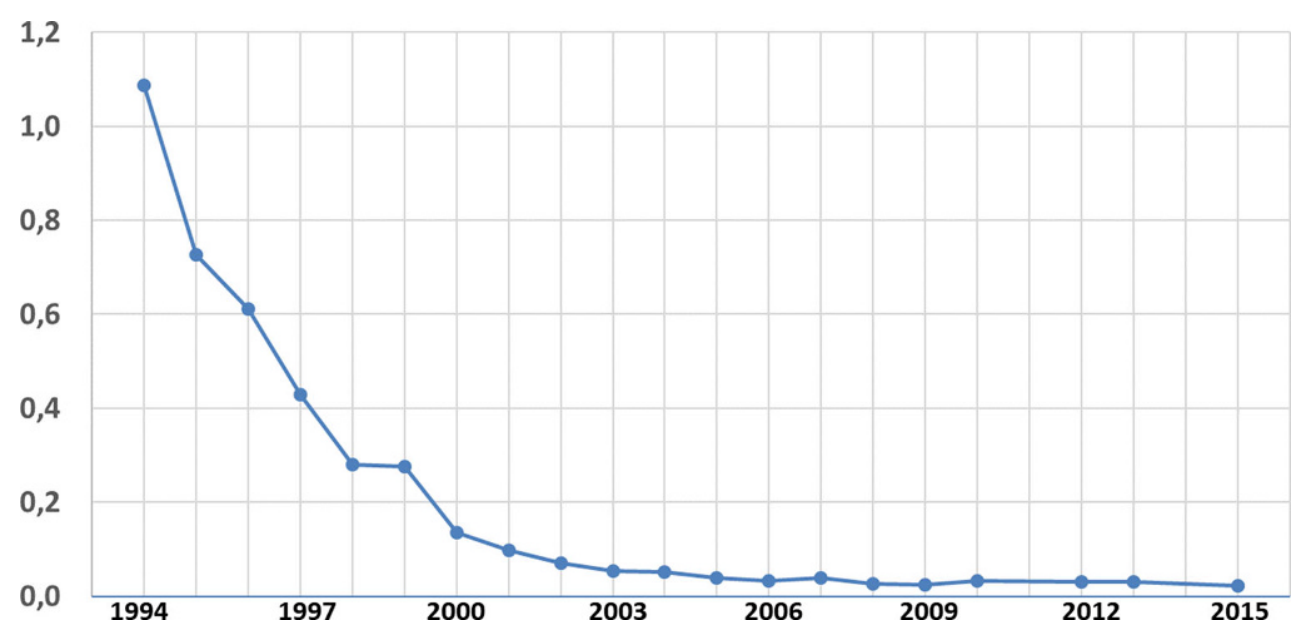

derten die Ausbildung „therapiefeindlicher Strukturen“ in den Einrichtungen.

Informiert man sich anhand des „Kerndatensatz im Maßregelvollzug“ ${ }^{\text {“3 }}$ über die diesbezüglichen Verhältnisse in den Kliniken, so findet man im Tabellenband der aktuellen Ausgabe u.a. folgende Daten: In den Jahrgängen 2010-2017 bewegte sich der Anteil der Patienten mit einer „Suchterkrankung“ nach Einschätzung der Behandler recht konstant im Bereich von 90\%. Für den Jahrgang 2017 wurde der Anteil der Patienten mit der ausschließlichen Diagnose „Persönlichkeitsstörung“ auf $0,5 \%$ geschätzt. Bei $17,8 \%$ wurde die Doppeldiagnose Sucht und Persönlichkeitsstörung gestellt. (Die Summe von über $100 \%$ resultiert aus der Möglichkeit von Mehrfachankreuzungen.) Die Stichtagserhebung beschreibt eine ähnliche Situation mit erheblichem Rückgang der Persönlichkeitsstörungsdiagnosen von 1994 bis 2014, allerdings auf einem etwas höheren Niveau (Riedemann und Berthold 2017).

Diese Zahlen stehen zwar im Widerspruch zu Müllers Darstellung, doch sind seine kritischen Hinweise auch nicht aus der Luft gegriffen. Persönlichkeitsstörungen werden in den Einrichtungen erheblich unterdiagnostiziert (Schalast et al. 2016). Das ist für die Praxis nicht unbedingt von Nachteil: Es bedarf in der forensischen Psychiatrie keiner die therapeutische Beziehung belastenden Anhäufung diagnostischer Zuschreibungen, um längere Behandlungszeiten zu rechtfertigen. Der Rückgang der Persönlichkeitsstörungsdiagnosen resultiert v. a. aus der Stabilisierung des Klinikalltags (Abb. 1), nicht daraus, dass inzwischen weniger problematische Patienten zur Aufnahme kämen. In den 1980er-Jahren waren die Behandler - aufgrund der häufi-

\footnotetext{
3 Jaschke H, Oliva H (2019) Kerndatensatz im MRV. Teil 2: Tabellenband 2017. Die Ausgaben des „Kerndatensatz im MRV“ sind nicht frei zugänglich, liegen jedoch den Trägern des Maßregelvollzugs, den Aufsichtsbehörden und den kooperierenden forensisch-psychiatrischen Einrichtungen vor.
}

gen problematischen Vorkommnisse - mit der Instabilität, Reizbarkeit und Süchtigkeit ihrer Patienten beständig konfrontiert.

Das NRW-Gesundheitsministerium ging Ende der Nullerjahre das Risiko ein, eine Evaluation zu finanzieren, mit der der Ertrag der Unterbringung einer strengen Prüfung unterzogen wurde (Schalast 2019; Schalast et al. 2019). In 16 Maßregelkliniken, darunter zur Hälfte Einrichtungen anderer Bundeslänger, wurden ab Ende 2009 konsekutiv Daten über alle neu aufgenommenen Patienten erhoben. Nach einem Merkmalsschema wurde zu jedem Patienten ein Vergleichsfall (Zwilling) im Strafvollzug rekrutiert. Es konnte gezeigt werden, dass der spätere Bewährungsverlauf der Patientenstichprobe signifikant günstiger war als der der Gefangenen, und zwar in der Gesamtbilanz, also bei Einbeziehung der Patienten mit ungünstigem Therapieverlauf. Die Effektgrößen waren deutlich höher als für die Behandlung von Straftätern und insbesondere auch Therapien im geschlossenen Vollzug üblicherweise berichtet.

\section{Zwischenbilanz}

Müller (2019) hat ein ausgesprochen negatives Bild der Entziehungsanstalten gemäß $\S 64$ StGB gezeichnet: eher kriminelle als kranke Menschen, zunehmend negative Verläufe, spektakuläre Vorkommnisse und Ausbrüche und geringe therapeutische Ansprechbarkeit. Bei Bezugnahme auf publizierte Daten relativiert sich das Bild, und es lassen sich durchaus auch positive Entwicklungen im Bereich der forensischen Suchtbehandlung aufzeigen.

Doch ist Müller insofern beizupflichten, als in den Kliniken eine besonders schwierige Patientengruppe behandelt wird, die so gar nicht der Wunschklientel vieler Psychotherapeuten entspricht. Die ist kompliant, motiviert, introspektiv und absprachefähig (und in der ambulanten Praxis überwiegend weiblich). Dagegen aktualisieren sich die Ent- 
wicklungs- und Persönlichkeitsstörungen der forensischen Patienten in der sozialen Interaktion (Streeck 2002), was die Arbeit mit ihnen anstrengend macht. Damit souverän und therapeutisch zielführend umzugehen, ist eine Herausforderung.

Die Kritik, dass - auch - „falsche“ Patienten kommen, hat eine gewisse Berechtigung. Die Fallzahlen erhöhen sich kontinuierlich, und der Aufnahmedruck ist aktuell enorm. Wegen des geringen Ermessensspielraums scheinen Gerichte die Unterbringung nicht selten entgegen ihrer Überzeugung und ihrem „Bauchgefühl“ anzuordnen, nur um die Rechtskraft des Urteils sicherzustellen (Kollmeyer 2013; zitiert nach Schalast 2017, Fn 18). Das ist ein Missstand und mit dem Ziel des verantwortlichen Umgangs mit therapeutischen Ressourcen schwer zu vereinbaren.

\section{Rechtslage und Reformbedarf}

Im Hinblick auf den gesetzlichen Reformbedarf ist Abb. 2 aufschlussreich: Rechtsreformen der Jahre 2007 und 2016 haben sich auf die Inanspruchnahme der Entziehungsanstalt offensichtlich erheblich ausgewirkt, aber nicht in erwünschter Weise: Die Reformen traten jeweils in Kraft, nachdem der Anstieg der Unterbringungszahlen sich über einige Jahre konsolidiert und ein Plateau erreicht hatte. Beide Reformen stießen jeweils einen erneuten Anstieg der Unterbringungszahlen an, insgesamt in der Größenordnung einer Verdoppelung. Dabei hatte zumindest die erste Reform das explizite Ziel, „die Kapazitäten des Maßregelvollzugs besser und zielgerichteter zu nutzen“ (BT-Dr. 16/1110, S. 9). Daraus ergibt sich für erneute Debatten über eine Reform des Maßregelrechts zwingend eine Priorität: Vor einer weitgehenden, in den Auswirkungen schwer einschätzbaren Umgestaltung aller sonstigen Unterbringungsvoraussetzungen müssen v. a. die zuletzt erfolgten Fehlregulierungen korrigiert werden.

Die Unterbringung ist bei Drogentätern mit höherer Straferwartung $\mathrm{zu}$ einem klaren Verteidigungsziel geworden, und zwar aufgrund des so erreichbaren Strafbzw. Vollzugsrabatts. Dies ist eine Folge der im „Gesetz zur Sicherung der Unterbringung in einem psychiatrischen Krankenhaus und in einer Entziehungsanstalt" vom 16.07.2007 getroffenen Regelungen, v.a. der Regelung zum Vorwegvollzug eines Teils der Strafe (damit nach einer angemessenen Therapiedauer die Entlassung in die Freiheit erfolgen kann). Die Krux der Bestimmung versteckt sich in Satz 3 des $\S 67$ Abs. 2: „Dieser Teil der Strafe ist so zu bemessen, dass nach seiner Vollziehung und einer anschließenden Unterbringung eine Entscheidung nach Absatz 5 S. 1 möglichst ist“. Letzterer besagt, dass bei diesen Patienten eine Vollstreckung des Strafrechts zur Bewährung ausgesetzt werden kann, wenn unter Anrechnung der Unterbringungszeit die Hälfte der Strafe erledigt ist. Diese Implikation wurde im Gesetzgebungsverfahren möglicherweise schlicht übersehen. Auf jeden Fall wurden die naheliegenden Auswirkungen nicht bedacht. Auch keiner der vom Rechtsausschuss gehörten Sachverständigen meldete Bedenken an!
Abb. 2 Entwicklung der Inanspruchnahme der Entziehungsanstalt (Zahl der jährlichen Neuanordnungen) in Relation zu zwei Gesetzesreformen. N.B.: Der Anstieg im Jahre 2007 ging zunächst auf die Einbeziehung der neuen Bundesländer (NBL) in die Strafverfolgungsstatistik zurück

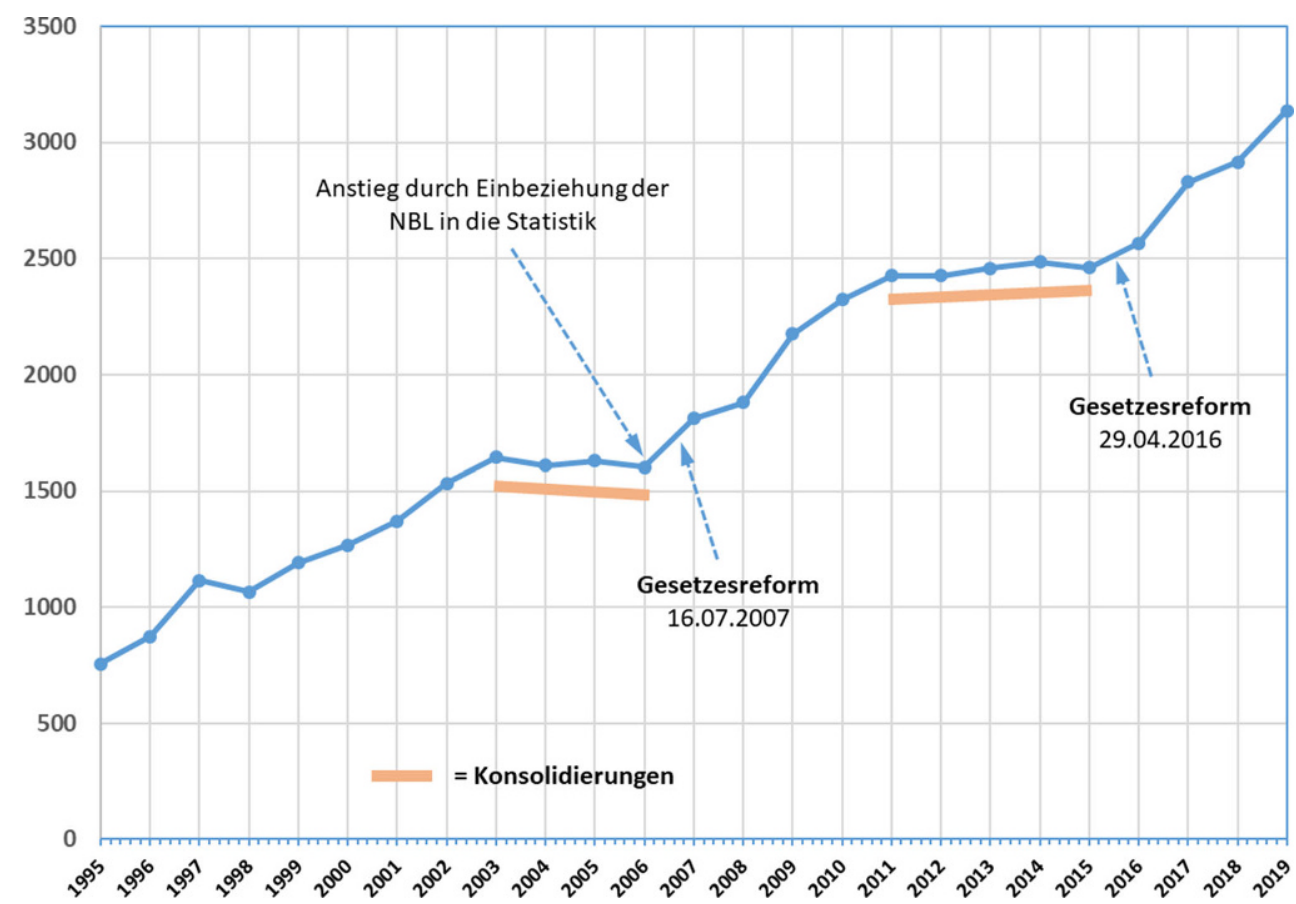


Die Folge ist, dass bei einem Drogentäter mit einer Straferwartung von erheblich über 3 Jahren die Anordnung der Unterbringung quasi der Hauptgewinn ist. Kollmeyer (2013) prägte die Formel: „Bis zwei Jahre Freiheitsstrafe will sie keiner, ab drei Jahre wollen sie alle, und zwar zunehmend mit der Höhe der Strafe“. Das Ziel, „,ie Kapazitäten des Maßregelvollzugs besser und zielgerichteter zu nutzen", wurde so konterkariert. BGH-Senate leisteten Schützenhilfe, indem sie Urteile reihenweise kassierten, in denen die Anwendung des § 64 StGB unterblieben war (Basdorf et al. 2011; im Überblick: Schalast 2017).

Die Situation wurde mit dem am 29.04.2016 verabschiedeten „Gesetz zur Novellierung des Rechts der Unterbringung in einem psychiatrischen Krankenhaus nach $\S 63$ StGB und zur Änderung anderer Vorschriften“ weiter verschlimmert. Bisher hatten sich die Gerichte bei der Berechnung des Vorwegvollzugs an $\S 67 \mathrm{~d}$ Abs. 1 Satz 1 orientiert: „Die Dauer der Unterbringung in einer Entziehungsanstalt darf zwei Jahre nicht übersteigen." Der Verfasser hatte in dieser Zeitschrift (Schalast 2013) nachdrücklich empfohlen, bei der Bestimmung des Vorwegvollzugs an der Höchstgrenze von 2 Jahren festzuhalten, und dies eingehend begründet. Der Gesetzgeber folgte jedoch einer Argumentation von Trenckmann (2011). Sie bezog sich auf einen umfangreichen Datensatz aus einem Landgerichtsbezirk, in dem allerdings mehr als $85 \%$ (von über 1000) Patienten in der Unterbringung scheiterten. Nur wer sich sehr lange - auch während einer langen Dauerbeurlaubung - als stabil erwiesen hatte, erhielt die Chance einer Entlassung zur Bewährung. Daraus wurde der verfehlte Kausalschluss gezogen: Nur sehr lange Behandlungen können erfolgreich sein. Leider überzeugte dies den Gesetzgeber. Wären die Zahlen Trenckmanns repräsentativ und die Praxis im betreffenden Landgerichtsbezirk alternativlos, so gäbe es nur eine Konsequenz: die Entziehungsanstalt gemäß § 64 StGB abzuschaffen.

In der Folge hat der Andrang auf die Entziehungsanstalten noch weiter zugenommen, was die Verantwortlichen trotz Ausweitung der Kapazitäten aktuell enorm unter Druck setzt. Einerseits kann der Verfasser sich Müllers Kritik nicht anschließen, es kämen auf diese Weise v.a. nichttherapiebedürftige Patienten in die Kliniken. Doch ist es tatsächlich ein Missstand, wenn Gerichte sich entgegen ihren Zweifeln an den Einlassungen des Beschuldigten genötigt sehen, die Unterbringung nur anzuordnen, damit das Urteil ,wasserdicht“" wird. Die Gerichte sollten einen konkreten Ermessensspielraum haben, wie er mit der Reform des Jahres $2007-\S 64$ StGB wurde als Sollregelung konzipiert - eigentlich intendiert war.

\section{Überlegungen zum Reformbedarf}

\section{Abschaffung der Entziehungsanstalten?}

Müller (2019) diskutiert zunächst, ob die Unterbringung gemäß $§ 64$ StGB nicht verzichtbar sei (S. 265). Dies bedarf keiner eingehenden Erörterung. Zum einen wird der Gesetzgeber einem solchen Vorschlag nicht folgen. Zum anderen wäre es auch nicht im allgemeinen Interesse, leidlich gut funktionierende Behandlungsstrukturen für eine Gruppe schwieriger Straffälliger abzuschaffen, für deren rehabilitativen Ertrag es einen belastbaren Nachweis gibt.

\section{Abhängigkeitssyndrom statt Hang?}

Müllers weitere Argumentation betrifft insbesondere die Art des Zusammenhangs zwischen Substanzgebrauch und Straffälligkeit und die Qualität der Abhängigkeitsproblematik, welche die Voraussetzung einer Unterbringung sein sollte. Hier fordert er insbesondere, den Begriff des Hangs in § $64 \mathrm{StGB}$,durch den medizinischen Begriff einer Abhängigkeitserkrankung im Sinne der jeweils gültigen (gegenwärtig ICD-10; Tab. 3) bzw. der zumindest mittelschweren Alkohol- bzw. Drogenkonsumstörung im Sinne des Diagnostic and Statistical Manual of Mental Disorders (Tab. 4, gegenwärtig DSM-5; Falkai et al. 2015)“ zu ersetzen (Müller 2019, S. 267).

Damit würde erreicht, ,dass für die angebotene Behandlung aus psychiatrischer Sicht auch eine Indikation besteht. ... Im Zweifel könnten die Angaben des Betroffenen zu ihrem Drogenkonsum durch zusätzliche Untersuchungen untermauert werden, z. B. könnten Blutuntersuchungen Leber- bzw. Blutveränderungen erfassen; damit könnte die Einnahme der relevanten Substanzen im Rahmen der jeweiligen Zeitfenster nachgewiesen werden. Eine Haaranalyse kann den Konsum auch über einen längeren Zeitraum untermauern.“

Hier ist anzumerken, dass solchen Untersuchungen auch nach geltendem Recht nichts entgegensteht. Eine „Indikation" besteht für die Unterbringung, wenn sie geeignet ist, die Gefahr erneuter hangbedingter Straftaten zu reduzieren. Ein schweres Abhängigkeitssyndrom ohne therapeutische Erfolgsaussicht wäre keine Indikation. Nach Kollmeyer (2013) ist allerdings gerade die Überprüfung der tatsächlichen Voraussetzungen der Anordnung, auch im Hinblick auf das Ausmaß der Suchtmittelproblematik, in entsprechenden Verfahren regelmäßig schwierig. So würden u. U. Konsummengen behauptet, die schon zum Zeitpunkt des Tätigwerdens eines Sachverständigen kaum noch objektivierbar, aber eben auch nicht widerlegbar sind.

Durch den Austausch der Begrifflichkeit - Hang gegen Abhängigkeitsdiagnose - wäre wenig gewonnen. Dies bestätigen auch die oben zitierten Daten der Stichtagserhe- 
bungen. Jahrzehntelang wurde immer wieder über die $\mathrm{Si}$ tuation der Entziehungsanstalten geklagt, aber nie darüber, dass die Patienten nicht süchtig genug wären. Außerdem ist der Rechtsbegriff des „Hangs“ in der geltenden BGH-Auslegung im Prinzip gut geeignet, Gelegenheitskonsumenten von Personen zu unterscheiden, deren Substanzkonsum hochproblematisch ist ${ }^{4}$. Zudem gibt es keinen Grund zu der Hoffnung, dass die Entziehungsanstalten (noch) erfolgreicher arbeiten könnten, wenn nur körperlich Schwerstabhängige untergebracht würden. Nochmals: Das Problem ist nicht der Begriff des Hangs, sondern sind die Schwierigkeiten, die tatsächlichen Voraussetzungen einer Anordnung verlässlich festzustellen. Die Rechtsreformen in 2007 und 2016 haben für Täter mit höherer Straferwartung einen erheblichen Anreiz geschaffen, diese Voraussetzungen zu konstruieren und mit ihren Einlassungen auf die Unterbringung hinzuwirken.

\section{Eine „Sollregelung“ für die Entziehungsanstalt, die ihren Namen verdient}

Mit der Maßregelrechtsreform vom 16.07.2007 wurde $\S 64$ StGB als Sollregelung gestaltet. Dies hat die Fachwelt nachdrücklich begrüßt. Auch dem Verfasser war damals nicht klar, dass eine solche Regelung rechtlich eine sehr verbindliche Vorschrift ist, der nur ausnahmsweise und wohlbegründet nicht gefolgt werden darf. Es blieb bei der seit Jahrzehnten bestehenden Tendenz der Obergerichte, die Nichtanordnung der Maßregel zu beanstanden und angefochtene Urteile aufzuheben (Basdorf et al. 2011).

Der ohnehin geringe Ermessensspielraum der Gerichte wurde durch Kommentierungen minimiert (Schalast 2017). Das galt auch für $\S 246$ a StPO, der die Einbeziehung von Sachverständigen in entsprechende Verfahren regelt. Im Hinblick auf $§ 64$ StGB als „Sollvorschrift“ war 2007 eine Differenzierung erfolgt: Der oder die Sachverständige ist zu hören, wenn a) die Unterbringung im psychiatrischen Krankenhaus in Betracht kommt oder b) das Gericht erwägt, die Unterbringung in einer Entziehungsanstalt anzuordnen. Kommentierungen haben dies nivelliert, nach dem Prinzip „kommt in Betracht, dann ist auch zu erwägen“ (Schneider 2008). Der Verfasser hat gemeinsam mit Lindemann (Schalast und Lindemann 2015) vorgeschlagen, den Ermessensspielraum durch die Einfügung eines Wortes in Satz 2 des $§ 246$ a Satz 2 StPO zu substanziieren: „Gleiches gilt,

\footnotetext{
${ }^{4}$ So heißt es im Strafrechtskommentar Schönke/Schröder (30. Aufl., 2019, § 64 Rz. 5): „Einen solchen Hang hat eine Person dann, wenn eine chronische, auf körperlicher Sucht bestehende Abhängigkeit oder zumindest eine eingewurzelte, auf psychischer Disposition bestehende oder durch Übung erworbene intensive Neigung besteht, immer wieder Alkohol oder andere Rauschmittel im Übermaß zu sich zu nehmen [...], also psychische Abhängigkeit besteht [...].“
}

wenn das Gericht konkret erwägt, die Unterbringung in einer Entziehungsanstalt anzuordnen.“

\section{Bezugnahme der Berechnung des Vorwegvollzugs auf den Zwei-Drittel-Zeitpunkt}

Um die Enthospitalisierung der vielen unter katastrophalen Umständen im „psychiatrischen Krankenhaus“ (§ 63 StGB) Verwahrten zu erleichtern, wurde bei der Großen Strafrechtsreform (2. StrRG, 01.01.1975) festgelegt, dass eine Entlassung schon erfolgen kann, wenn noch nicht zwei Drittel der Strafe erledigt sind. 1986 (23. StrÄndG) wurde präzisiert, dass die Aussetzungsmöglichkeit besteht, wenn unter Anrechnung verbüßter Haft die Hälfte der Strafe erledigt ist. Wohl mehr oder weniger unbeabsichtigt wurde diese Bestimmung bei der Reform des Jahres 2007 auch auf die Entziehungsanstalt bezogen und durch die Regelung zum Vorwegvollzug ins Bewusstsein aller Beteiligten gerückt.

In der Fachwelt besteht Übereinstimmung, dass die Berechnung sich bei Anwendung von $\$ 64$ StGB auf den ZweiDrittel-Zeitpunkt beziehen muss, um keine Fehlanreize zu schaffen. Dies vertritt auch Müller (2019, S. 269).

\section{Verzicht auf Anordnung der Unterbringung zugunsten der Perspektive einer Behandlung im Rahmen einer Strafzurückstellung (§ 35 BtMG)}

Wie an anderer Stelle (Schalast 2017, S. 438) eingehend begründet und in der Vergangenheit vielfach gefordert, sollte in konkreten Einzelfällen eine Möglichkeit verbleiben, zugunsten der Perspektive einer späteren Therapie im Rahmen einer Strafzurückstellung ( $\$ 35$ BtMG) von der Anordnung der Unterbringung in der Hauptverhandlung abzusehen. Dies sollte, so Basdorf et al. (2011, S. 62), z. B. Anerkennung finden, wenn die zeitlichen Gegebenheiten für eine Zurückstellung in absehbarer Zeit erfüllt sind, der Angeklagte eine entsprechende Motivation offenbart und die tatsächliche Realisierbarkeit vorliegt. Entsprechende Anregungen blieben jedoch ohne eine Konkretisierung im Gesetz bisher stets folgenlos. Es wird daher vorgeschlagen, § 64 Abs. $1 \mathrm{StGB}$ in etwa folgender Weise zu ergänzen:

Die Unterbringung in einer Entziehungsanstalt kann unterbleiben, wenn eine Suchtbehandlung im Rahmen einer Strafzurückstellung im Vollstreckungsverfahren realisierbar ist und ebenso erfolgversprechend erscheint. 


\section{Vorläufige bzw. befristete Unterbringung zur Behandlungserprobung?}

Wie schon Querengässer et al. (2016) empfiehlt auch Müller (2019, S. 269) eine Art vorläufiger Unterbringung oder Probetherapie. In dieser Zeit solle mit dem Patienten Motivationsarbeit geleistet und seine Therapiebereitschaft und -eignung und sein Verhalten im Kontext mit anderen Patienten beobachtet werden. Auf dieser Basis könne dann fundierter zur Prognose Stellung genommen und der Therapieerfolg vorhergesagt werden. Verwiesen wird auf eine frühere Untersuchung, publiziert unter dem Titel „Motivation oder Aussichtslosigkeit“" (Müller 2005). Mit dieser Studie kann der Nutzen des Prinzips „Probetherapie“ nicht gut begründet werden. Unter anderem sei eine relativ niedrige Erledigungsquote erreicht worden, doch wurden die vollständigen Therapieverläufe zum Zeitpunkt der Publikation noch gar nicht überblickt.

Jede Therapeutin wünscht sich „motivierte“ Patienten. Gerade manchen primär dissozialen Patienten mit weniger pathologischer Delinquenz (Eigentum, BtMG, Gewalt im Milieu) fällt es leicht, therapeutische Erwartungen (oberflächlich) zu erfüllen. Insbesondere Patienten mit beschämenden Delikten (Sexualtaten, häusliche Gewalt) können massiv unter Druck geraten, ,,sperren sich“ - gerade bei Konfrontation im Gruppensetting, weil sie Beschämung und soziale Ausgrenzung fürchten - und sind doch oft besonders therapiebedürftig.

Der § 64-Maßregelvollzug hat unter den gegenwärtigen schwierigen Bedingungen eine beachtliche rehabilitative Wirksamkeit entwickelt. Hätten die Gerichte einen größeren Ermessensspielraum, so würde die Problematik völlig deplatzierter Patienten ohnehin abgemildert. Das Prinzip Probetherapie könnte einen „Verschiebebahnhof“" eröffnen, dessen Dimensionen nicht kalkulierbar sind.

\section{Orientierung an der in § 67d StGB genannten zeitlichen Höchstgrenze für die Unterbringung bei der Berechnung des Vorwegvollzugs}

Der Verfasser rät dringend dazu, bei der Berechnung des Vorwegvollzugs von Strafe - wie vor dem Reformgesetz des Jahres 2016 - von der Höchstgrenze von 2 Jahren auszugehen. Dieser Begrenzung lag ursprünglich die Überlegung zugrunde, dass diese Maßregel in erster Linie als bessernde Maßnahme zu verstehen und nur innerhalb einer bestimmten Frist sinnvoll und erfolgversprechend sei (so Hanack im Leipziger Kommentar ${ }^{5}$ ). Rasch (1991) vertrat die Auffassung, dass realistischerweise eine länger als 2 Jahre dauernde Entwöhnungsbehandlung kaum durchführbar sei. Das

5 LK/Hanack, StGB, 10. Aufl., § 67d Rn. 2, vgl. a. LK/Schöch, StGB, 12. Aufl., § 64 Rn. 167. trifft sicher zu, wenn man von der geschlossenen stationären Therapiezeit ausgeht.

In NRW waren die Unterbringungszeiten über Jahre besonders hoch. Dies beruhte auch darauf, dass das Maßregelvollzugsgesetz des Landes lange Dauerbeurlaubungen aus der Unterbringung ermöglicht. Es spielte sich ein, dass Patienten erst nach sehr langer Bewährung im Rahmen der Beurlaubung formell entlassen wurden. Dies hatte u. a. zur Folge, dass die Gesamtunterbringungszeiten bis zur Entlassung in die Freiheit in NRW doppelt so hoch waren wie in Bayern (Schalast 2013, Tab. 1). Leider war gleichzeitig die Quote erfolgreicher Behandlungsverläufe in NRW mancherorts katastrophal niedrig. Dazu können überlange Unterbringungs- und Beurlaubungszeiten sogar beigetragen haben.

\section{Zur Frage der Kausalbeziehung zwischen Suchtproblemen und Straffälligkeit}

Im Rahmen eines Diskussionsbeitrags erklärte eine Vertreterin der forensischen Psychiatrie, die Unterbringung solle bei Personen erfolgen, deren Straffälligkeit sich eindeutig - gleichsam monokausal - als Folge der Suchtproblematik darstelle. In der sozialen Wirklichkeit sind Straffälligkeit und Drogenkonsum insbesondere bei jungen Menschen parallele Verhaltensprobleme, für die ähnliche Risikofaktoren bedeutsam sind (Mulvey et al. 2010). Die beiden Problembereiche können sich wechselseitig verstärken, und Suchtprobleme erschweren das Herauswachsen aus einer dissozialen Entwicklung (Hussong et al. 2004; Kreuzer 2015). Die Frage, ob ein Patient nicht „eigentlich“ dissozial ist, ist wenig zielführend. In der Therapie geht es darum, konstruktive Perspektiven zu entwickeln und einen impulsiven, fremd- und selbstschädigenden Verhaltensstil zu überwinden.

Es entspricht allerdings der Logik einer „Unterbringung in der Entziehungsanstalt", dass die Straffälligkeit in den Suchtproblemen eine wesentliche Ursache hat. Das sollte auch in $\S 64$ StGB entsprechend zum Ausdruck kommen. Die Formulierung würde die Nichtanordnung bei Tätern rechtfertigen, für deren Delinquenz Suchtprobleme von eindeutig nachrangiger Bedeutung sind. Auch dies würde den Ermessensspielraum der Gerichte erweitern. Es ist aber hinzunehmen, dass im Einzelfall praktisch immer weitere wichtige ursächliche Faktoren hinzukommen (gewaltaffines Elternhaus, Hyperaktivitätsprobleme in der Kindheit u. a.m.). Die Formulierung „,die wesentliche Ursache“ wäre insofern geeignet, viele für eine Behandlung empfängliche Täter mit Suchtproblemen von vorneherein auszuschließen. Die Änderung würde $\S 64$ Satz 1 betreffen: „Hat eine Person den Hang, alkoholische Getränke oder andere berauschende Mittel im Übermaß zu sich zu nehmen und wird sie wegen einer rechtswidrigen Tat, die sie im Rausch begangen hat 
oder die in ihrem Hang eine wesentliche Ursache hat, verurteilt ...".

\section{Knüpfung der Unterbringung an die Feststellung einer mindestens verminderten Schuldfähigkeit?}

Es ist bemerkenswert, dass Gesetzeslage und Rechtsprechung eine große Affinität zur Anordnung der Behandlungsmaßregel erkennen lassen, während sie gleichzeitig sehr bemüht sind, gegenüber der Dekulpierung aufgrund von Suchtproblemen eine hohe Schwelle aufrechtzuhalten. So heißt es in einem BGH-Urteil (BGH 1 StR 15/12 vom 17.04.2012): „Die bloße Abhängigkeit (von Drogen) beeinflusst für sich genommen die Steuerungsfähigkeit (...) nicht. Dies ist erst dann in Erwägung zu ziehen, wenn langjähriger Betäubungsmittelmissbrauch zu schwersten Persönlichkeitsveränderungen geführt hat. In diesen Fällen liegen regelmäßig zugleich ein organischer Befund und eine krankhafte seelische Störung vor. Auch beim akuten Rausch ist ein Ausschluss oder die erhebliche Verminderung der Steuerungsfähigkeit möglich. Schwere Entzugserscheinungen können die Steuerungsfähigkeit bei Beschaffungsdelikten nur in seltenen Ausnahmefällen, z.B. in Kombination mit Persönlichkeitsveränderungen, aufheben.“

Dass strafrechtliche Verantwortlichkeit und Therapiebedürftigkeit so gänzlich voneinander abgekoppelt sind, ist eigentlich sonderbar. Dennoch sollte eine Einschränkung der Schuldfähigkeit nicht zur Voraussetzung der Unterbringung gemacht werden. Suchtprobleme sind ein Risikofaktor für delinquente Rückfälligkeit. Es erscheint nicht vertretbar, mit einem ernsthaften Behandlungsversuch grundsätzlich abzuwarten, bis die im oben genannten BGH-Urteil beschriebenen Folgeschäden eingetreten sind. Auch das würde die Anwendung des $\S 64$ StGB auf eine prognostisch besonders problematische Gruppe beschränken.

\section{Zur hohen Quote von Erledigungen der Unterbringung}

Man macht es sich leicht, wenn negative Behandlungsverläufe stets mit der „Fehlplatzierung“ der betroffenen Untergebrachten erklärt werden. Kröber (2020) kommentierte in dieser Zeitschrift unlängst kritisch die Tendenz, „dass der Therapeut und die Klinik immer recht haben, falls es zu Konflikten kommt" (Kröber 2020, S. 117). Eine Analyse zeigte, dass die „Erledigungsquoten“ der einzelnen Kliniken über die Jahre eine gewisse Konstanz aufweisen. Das heißt, eine hohe Quote im Jahr X lässt auch eine überdurchschnittliche Quote in den Folgejahren erwarten (Schalast 2019, S. 112f.). Dies kann natürlich nicht mit Patientenmerkmalen erklärt werden; in manchen Kliniken scheint therapeutisch angemessener gearbeitet zu werden als in an- deren. Eine eher niedrige Erledigungsquote sollte als Qualitätsmerkmal der Arbeit forensischer Abteilungen anerkannt werden.

Dabei könnte auch der Aufnahmedruck, unter dem Kliniken stehen, eine Rolle spielen. Die Toleranz der Kliniken im Umgang mit Problemverhalten der Patienten ist bei niedrigem Aufnahmedruck tendenziell höher, während bei hohem Aufnahmedruck schneller mit einem Antrag auf Erledigung reagiert wird. $\mathrm{Zu}$ diesem einerseits verständlichen, andererseits hochproblematischen Sachverhalt wurden bisher keine spezifischen Daten publiziert. Bei Müller (2005) und Pollähne und Kemper (2007) finden sich entsprechende Hinweise.

\section{Fazit}

Auch infolge der Maßregelrechtsreformen der Jahre 2007 und 2016 hat sich der Aufnahmedruck, unter dem die Entziehungsanstalten stehen, weiter erhöht. Für Angeklagte mit hoher Straferwartung wurden starke Anreize geschaffen, sich um die Unterbringung zu bemühen. Strafgerichte haben - bei entsprechenden Einlassungen der Beschuldigten geringe Ermessensspielräume, die Unterbringung revisionssicher abzulehnen.

Dies hat zu einem stetigen Anstieg der Unterbringungszahlen beigetragen, der leider auch mit einem beträchtlichen Anteil negativer Verläufe einhergeht. Patienten, die nach ungünstigem Verlauf in die Strafhaft zurückverlegt werden, profitieren - gemessen an ihren Bewährungsquoten - von monatelangen Behandlungsbemühungen überhaupt nicht.

Die erkennenden Gerichte bedürfen hinsichtlich der Anwendung des $§ 64$ StGB eines realen Ermessensspielraums. Der Beitrag macht Vorschläge, wie dieser konkretisiert werden kann.

Der mit dem Reformgesetz verbindlich eingeführte Vorwegvollzug eines Teils einer längeren Freiheitsstrafe sollte wieder auf den Zwei-Drittel-Zeitpunkt Bezug nehmen.

Mit dem Reformgesetz vom 29.04.2016 wurde die Möglichkeit eröffnet, bei der Bestimmung des Vorwegvollzugs von vorneherein von Therapiezeiten von über 2 Jahren auszugehen. Die Berechnung des Vorwegvollzugs muss sich wieder an der Höchstgrenze von 2 Jahren orientieren.

Gerichten sollte grundsätzlich ein Spielraum verbleiben, im Einzelfall von der Anordnung der Unterbringung zugunsten einer Behandlung im Rahmen einer Strafzurückstellung (§ 35 BtMG) abzusehen. Dies sollte auch im Gesetz zum Ausdruck kommen.

Der regelhaften Einführung einer Art Probetherapie, aus der heraus in den ersten Monaten schnell eine Rückverlegung in die Haft erfolgen kann, steht der Verfasser zwiespältig gegenüber. Die modernen Entziehungsanstalten ha- 
ben ihre Konzepte unter den schwierigen Bedingungen der letzten Jahrzehnte entwickelt und arbeiten mit Ertrag. Und hätten die Gerichte im erkennenden Verfahren einen reellen Ermessensspielraum, so würde manche fragwürdige Unterbringungsanordnung von vorneherein unterbleiben.

Funding Open Access funding enabled and organized by Projekt DEAL.

Interessenkonflikt N. Schalast gibt an, dass kein Interessenkonflikt besteht.

Open Access Dieser Artikel wird unter der Creative Commons Namensnennung 4.0 International Lizenz veröffentlicht, welche die Nutzung, Vervielfältigung, Bearbeitung, Verbreitung und Wiedergabe in jeglichem Medium und Format erlaubt, sofern Sie den/die ursprünglichen Autor(en) und die Quelle ordnungsgemäß nennen, einen Link zur Creative Commons Lizenz beifügen und angeben, ob Änderungen vorgenommen wurden.

Die in diesem Artikel enthaltenen Bilder und sonstiges Drittmaterial unterliegen ebenfalls der genannten Creative Commons Lizenz, sofern sich aus der Abbildungslegende nichts anderes ergibt. Sofern das betreffende Material nicht unter der genannten Creative Commons Lizenz steht und die betreffende Handlung nicht nach gesetzlichen Vorschriften erlaubt ist, ist für die oben aufgeführten Weiterverwendungen des Materials die Einwilligung des jeweiligen Rechteinhabers einzuholen.

Weitere Details zur Lizenz entnehmen Sie bitte der Lizenzinformation auf http://creativecommons.org/licenses/by/4.0/deed.de.

\section{Literatur}

Basdorf C, Schneider U, König P (2011) Vom Umgang des Revisionsgerichts mit $\S 64$ StGB. In: Fischer T, Bernsmann K (Hrsg) Festschrift Rissing-van Saan. De Gruyter, Berlin, S 59-74

Hussong AM, Curran PJ, Moffitt TE, Caspi A, Carrig MM (2004) Substance abuse hinders desistance in young adults' antisocial behavior. Develop Psychopathol 16(4):1029-1046

Kollmeyer R (2013) Hinreichend konkrete Voraussetzungen für eine erfolgreiche Behandlung aus rechtlicher Sicht? Präsentation und Vortrag bei der Fachtagung „Maßregelvollzug am Limit“ von LWL und OLG Hamm. http://www.lwl.org/massregelvollzugdownload/Abt62/Service/Dokumentationen/OLG-Tagung2013/ 2013-09-26_Kollmeyer,_Reinhard_OLG-Hamm_Para_64_ StGB_Wann_und_wie_lang.pdf. Zugegriffen: 25. März 2020

Kreuzer A (2015) Zusammenhänge zwischen Drogen und Kriminalität. Forens Psychiatr Psychol Kriminol 9:3-9

Kröber HL (2020) Der gemaßregelte Verurteilte. Forens Psychiatr Psychol Kriminol 14:117-118

Lehmann M (2019) Suchtprobleme bei Gefangenen: Situation und Perspektiven. In: Schalast N (Hrsg) Straffällige mit Suchtproblemen. Pabst, Lengerich, S 17-28

Müller JL (2005) Motivation oder Aussichtslosigkeit? Konzept und 1-Jahres-Evaluation einer suchtforensischen Aufnahme-, Diagnostik- und Motivationsstation. Psychiatr Prax 32:205-207

Müller JL (2019) Ansätze zur Reform der Unterbringung in einer Entziehungsanstalt. Forens Psychiatr Psychol Kriminol 13:262-271

Müller JL, Saimeh N, Briken P et al (2017) Standards für die Behandlung im Maßregelvollzug nach $\S \S 63$ und 64 StGB. Nervenarzt 88(Suppl 1):S1-S29
Mulvey EP, Schubert CA, Chassin L (2010) Substance use and delinquent behavior among serious adolescent offenders. https://www. ncjrs.gov/pdffiles1/ojjdp/232790.pdf. Zugegriffen: 28. Jan. 2021 (Juvenile Justice Bull.)

Pollähne H, Kemper A (2007) Fehleinweisungen in die Entziehungsanstalt. Bremer Forschungen zur Kriminalpolitik, Bd. 10. LIT, Berlin

Querengässer J, Ross T, Bulla J, Hoffmann K (2016) Neue Wege in die Entziehungsanstalt - Reformvorschläge für den $§ 64 \mathrm{StGB}$. NStZ 36:508-510

Querengässer J, Bulla J, Hoffmann K, Ross T (2017) Outcomeprädiktoren forensischer Suchtbehandlungen Teil II _ Zum Zusammenhang von personen- und nicht-personengebundenen Faktoren mit der Legalbewährung nach Entlassung aus einer Unterbringung nach § 64 StGB. Recht Psychiatr 35:139-146

Rasch (1991) Voraussetzungen der Unterbringung gemäß § 64 StGB aus psychiatrischer Sicht. Recht Psychiatr 9(3):109-114

Riedemann C, Berthold D (2017) Deutschlandweite Stichtagserhebung. www.mrvzn-badrehburg.niedersachsen.de/download/ 142274/Stichtagserhebung_2017.pdf. Zugegriffen: 23. Jan. 2021

Schalast N (1994) Unterbringung in der Entziehungsanstalt. Probleme der Behandlung alkoholabhängiger Straftäter. Recht Psychiatr 12:2-10

Schalast N (1999) Maßregelvollzug - Medien - Klinikprojekte. In: Wiegand W (Hrsg) Der Maßregelvollzug in der öffentlichen Diskussion. Votum, Münster, S 20-25

Schalast N (2013) Zur Dauer der Unterbringung in einer Entziehungsanstalt. Forens Psychiatr Psychol Kriminol 7(2):105-113

Schalast N (2017) Missbrauch der Entziehungsanstalt. NStZ 37: 433-439

Schalast N (2019) Ergebnisse der Essener Evaluationsstudie. In: Schalast N (Hrsg) Straffällige mit Suchtproblemen: Fakten, Erfahrungen und Ergebnisse der Essener Evaluationsstudie. Pabst, Lengerich, S 29-150

Schalast N, Lindemann M (2015) Anmerkungen zu den Plänen einer Änderung des Rechts der Unterbringung im psychiatrischen Krankenhaus. Recht Psychiatr 33:72-84

Schalast N, Frey M, Boateng S, Demmerling R, von der Haar M (2016) Persönlichkeitsstörungen - unterdiagostiziert bei Patienten des Maßregelvollzugs gemäß § 64 StGB? Sucht 62(5):305-313

Schalast N, Frey M, Boateng S, Dönisch-Seidel U, Leygraf N (2019) Was rechtfertigt eine Behandlungsmaßregel für Täter mit Suchtproblemen? Recht. Psychiatrie 37(3):141-146 (abrufbar von: https://forschen-und-teilen.de/wp-content/uploads/2019/06/RP_ 19_3_schalast-et-al.pdf)

Schneider U (2008) Die Reform des Maßregelrechts. NStZ 28:68-73

Streeck U (2002) Gestörte Verhältnisse - Zur psychoanalytisch-interaktionellen Gruppentherapie von Patienten mit schweren Persönlichkeitsstörungen. Persönlichkeitsstörungen 2:109-125

Trenckmann B (2011) Zur Verweildauer im Maßregelvollzug einer Entziehungsanstalt gemäß $§ 64$ StGB. NStZ 31:322-330

von der Haar M (2016) Stichtagserhebung im MRV nach § 64 StGB von 1994 bis 2015. https://www.mrvzn-badrehburg.niedersachsen. de/startseite/veroffentlichungen/veroeffentlichungen-106184. html. Zugegriffen: 13.06.2020

Zimprichová D (2019) Klinische Erfahrungen mit unterschiedlichen Gruppen drogenabhängiger Straftäter und mit Substitution. In: Schalast N (Hrsg) Straffällige mit Suchtproblemen. Pabst, Lengerich, S 29-150 